\title{
Primary malignant melanoma of the trachea: A case report
}

\author{
HISAO IMAI $^{1}$, YOSHIO KIYOHARA ${ }^{2}$, SHUSUKE YOSHIKAWA ${ }^{2}$, NAO KUSUTANI $^{2}$, AKIRA ONO $^{1}$, \\ TETSUHIKO TAIRA ${ }^{1}$, HIROTSUGU KENMOTSU ${ }^{1}$, HIDEYUKI HARADA ${ }^{3}$, \\ TATEAKI NAITO $^{1}$, HARUYASU MURAKAMI ${ }^{1}$, TAKEHISA SANO ${ }^{4}$, HIROSHI FUJI ${ }^{5}$, \\ MASAHIRO ENDO $^{6}$, TAKASHI NAKAJIMA ${ }^{7}$ and TOSHIAKI TAKAHASHI ${ }^{1}$
}

\begin{abstract}
Divisions of ${ }^{1}$ Thoracic Oncology, ${ }^{2}$ Dermatology and ${ }^{3}$ Radiation Oncology, Shizuoka Cancer Center, Suntou-gun, Shizuoka 411-8777; ${ }^{4}$ Department of Respiratory Medicine, Shizuoka City Hospital, Aoi-ku, Shizuoka 420-8630; Divisions of ${ }^{5}$ Proton Therapy, ${ }^{6}$ Diagnostic Radiology and ${ }^{7}$ Diagnostic Pathology,

Shizuoka Cancer Center, Suntou-gun, Shizuoka 411-8777, Japan
\end{abstract}

Received February 17, 2014; Accepted September 16, 2014

DOI: $10.3892 / 01.2014 .2782$

\begin{abstract}
Primary cancer of the trachea is rare and accounts for only $0.1-0.4 \%$ of all newly diagnosed respiratory tract cancers, worldwide. In the present study, a case of primary tracheal malignant melanoma, a particularly rare type of cancer, is reported. A 68-year-old male presented with a cough and bloody sputum. A chest computed tomography scan revealed a $25 \times 20 \times 15-\mathrm{mm}$ tracheal tumor, located immediately above the carina, which reduced the cross-sectional area of the trachea by $\sim 90 \%$. Histopathological analysis of biopsy specimens determined a diagnosis of malignant melanoma. The patient was treated with argon plasma coagulation and chemoradiotherapy, which restored airway patency, however, metastasis was detected in the lungs. The patient refused further treatment and received palliative care. Subsequently, the patient succumbed to the disease within four months. Thus, although primary malignant melanoma of the trachea is extremeley rare, the possibility should be considered during diagnosis.
\end{abstract}

\section{Introduction}

Primary cancer of the trachea is particularly rare (1) and accounts for only $0.1-0.4 \%$ of all newly diagnosed respiratory tract cancers, which corresponds to 2.6 new cases per 1,000,000 individuals, annually, worldwide $(2,3)$. Approximately $75 \%$ of these tumors are squamous cell carcinoma or adenoid cystic carcinoma (4). Malignant melanomas occur primarily as skin lesions and account for $2 \%$ of all

Correspondence to: Dr Hisao Imai, Division of Thoracic Oncology, Shizuoka Cancer Center, 1007 Shimonagakubo, Nagaizumi-chou, Suntou-gun, Shizuoka 411-8777, Japan

E-mail: m06701014@gunma-u.ac.jp

Key words: malignant melanoma, trachea, argon plasma coagulation skin tumors, worldwide (5). Although primary malignant melanoma frequently metastasize to the liver, lung, brain, or bone, this type of cancer rarely occurs in parts of the body other than the skin. The most uncommon form of extracutaneous melanoma is primary tracheal melanoma (6-10). In the present study, the case of a patient presenting with malignant melanoma of the trachea is reported to improve the current understanding of this rare disease. Written informed consent was obtained from the patient.

\section{Case report}

In March 2013, a 68-year-old male presented to the Department of Respiratory Medicine, Shizuoka City Hospital (Shizuoka, Japan) with a cough and bloody sputum, which had worsened over the previous two month and become intractable, with the development of stridor. A chest computed tomography (CT) scan revealed a $25 \times 20 \times 15-\mathrm{mm}$ intratracheal lesion located immediately above the carina (Fig. 1), which reduced the cross-sectional area of the trachea by $\sim 90 \%$. Flexible bronchoscopy demonstrated these results and revealed an obstructive tumor surrounding the carina, as well as irregularly shaped, darkened regions in the tracheal mucosa (Fig. 2A).

Following biopsy of the tumor and the surrounding mucosa, the tumor was cauterized with argon plasma coagulation (APC) to restore airway patency, however, the presence of a residual tumor mass was not clear. Following cauterization, the patient experienced immediate symptomatic relief. The biopsy specimens were composed of tumor fragments and aggregated melanophages. Histologically, hematoxylin and eosin staining of the tumor demonstrated proliferation of epithelioid-shaped atypical cells with marginal melanin production (Fig. 3A). In addition, the tumor cells showed positive immunostaining for three melanoma markers, S-100, melan-A and HMB-45 (Fig. 3B-D). Subsequently, the tumor was diagnosed as a malignant melanoma. The biopsy specimens from the bronchial mucosa revealed a band-like accumulation of melanophages and lymphocytes beneath the tracheal epithelium. However, melanoma infiltration was not observed among the melanophages. 


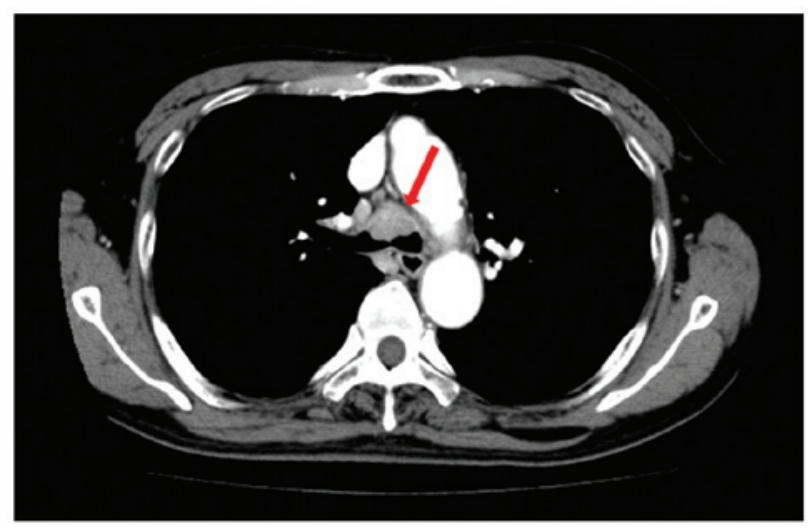

Figure 1. Chest computed tomography scan. The tumor measured 25x20x15 mm and was located directly above the carina and protruded from the anterior wall of the trachea (arrow).
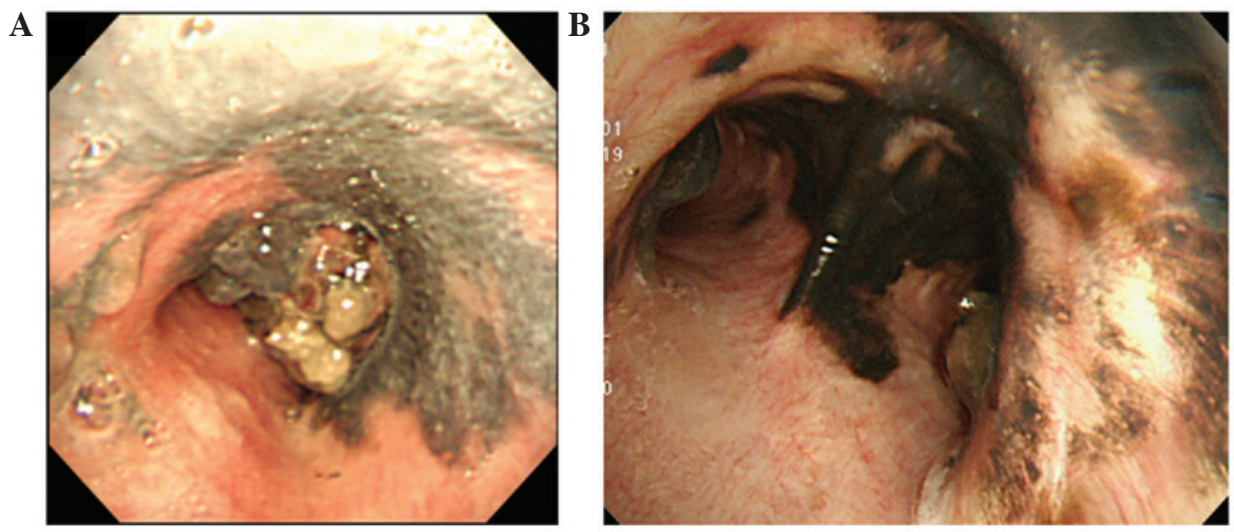

Figure 2. Endoscopic findings. (A) Prior to treatment, bronchoscopy revealed a pigmented, cauliflower-like, warty tumor. (B) Following chemoradiotherapy, bronchoscopy revealed darkened regions in the tracheal mucosa.

A

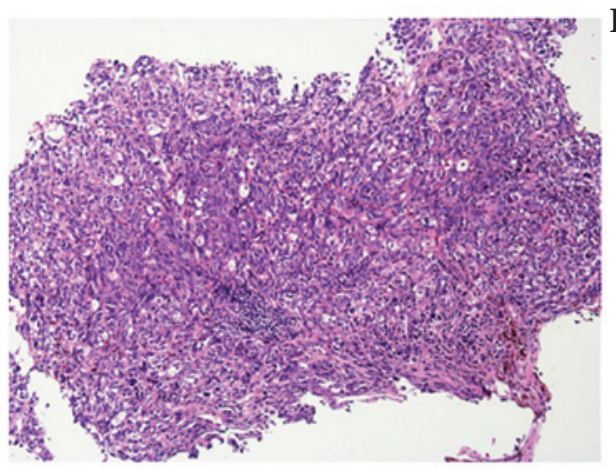

C

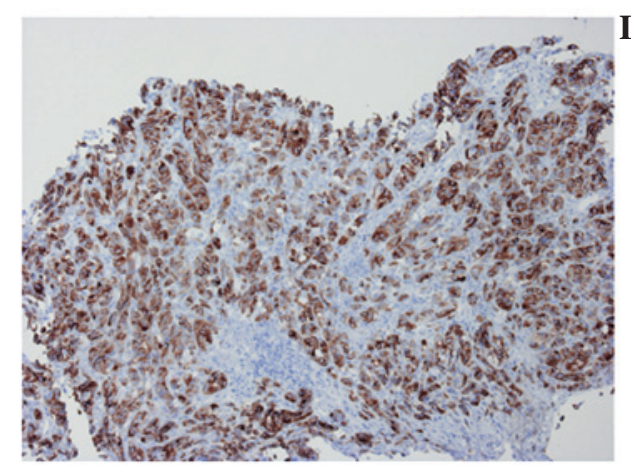

B
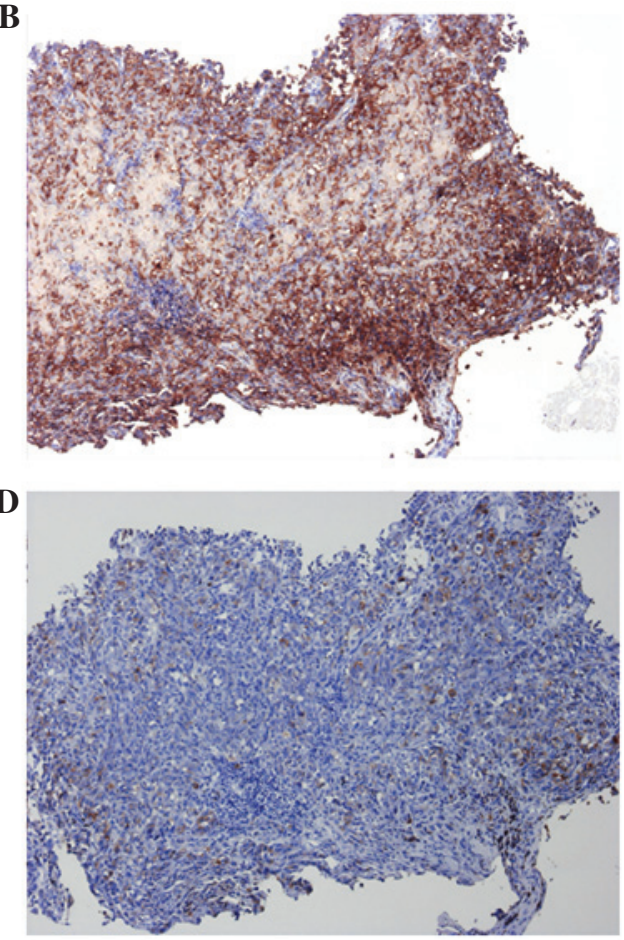

Figure 3. Histological and immunohistochemical observations of tumor tissue obtained via bronchoscopic biopsy. (A) Proliferation of epithelioid-shaped atypical cells with marginal melanin production were observed (stain, hematoxylin and eosin; magnification, x100). In the same tumor, these epithelioid-shaped atypical cells also stained positively for the melanoma markers (B) S-100, (C) melan-A and (D) HMB-45 (magnification, x100). 
The patient did not have a history of previous surgeries or skin biopsies and did not exhibit melanoma-like skin lesions. In addition, magnetic resonance imaging and positron emission tomography did not reveal any metastatic lesions in the brain. The level of 5-S-cysteinyldopa, a biochemical marker of melanoma, which was $7.6 \mathrm{nmol} / \mathrm{l}$ (normal range, 1.5-8.0 nmol/l) at diagnosis, did not increase. As a result of these findings, it was hypothesized that the trachea was the primary site of the tumor and no metastasis had occurred at the time of clinical presentation.

The lesion was inoperable due to its large size; therefore, the patient was treated with a combination of dacarbazine-based chemotherapy $\left(200 \mathrm{mg} / \mathrm{m}^{2}\right.$ dacarbazine, days 1-5) for three cycles every 4 weeks, for three months and thoracic radiotherapy (total dose, $65 \mathrm{~Gy}$ in 30 fractions). Following chemoradiotherapy, bronchoscopy revealed darkened regions of the tracheal mucosa (Fig. 2B). Subsequently, metastatic lesions appeared in the lungs and the 5-S-cysteinyldopa levels gradually increased, thus, chemotherapy was resumed.

\section{Discussion}

Primary tracheal malignant melanoma is particularly rare (6-10) and there are only a small number of reports regarding intratracheal metastasis (11). Various studies have investigated the oncogenesis of mucosal melanomas and have attempted to elucidate the histogenesis of lower respiratory tract melanomas $(12,13)$. Theories include melanocytic migration during embryogenesis, transformation of respiratory epithelial cells into melanocytes and differentiation of neuroendocrine cells to melanocyte (12).

Pathological examination cannot distinguish primary melanoma from metastatic melanoma. The criteria for primary respiratory malignant melanoma diagnosis are as follows: A solitary lesion; 'dropping off' of melanoma cells together with junctional changes in the mucosa; invasion from the epithelium toward the submucosa; histologically identified presence of melanin; no prior skin lesions; and no familial history of cutaneous disease (12). In the present case, no other primary lesions were identified on radiological or dermatological examination. The patient was diagnosed with a primary tracheal malignant melanoma on the basis of three criteria: The lack of a history of skin lesions and a family history of cancer; the presence of a solitary tumor surrounded by abnormal mucosa; and positive immunostaining for three melanoma markers, S-100, melan-A and HMB-45. These results are consistent with the diagnostic criteria for primary malignant melanoma in the respiratory tract $(13,14)$.

Tracheal tumors may be fatal as they occasionally obstruct the airway. However, due to their rarity, no standard treatment has been identified. Treatment is either palliative, which aims to restore airway patency, or therapeutic, with tracheal resection and end-to-end anastomosis (15). In the current case, APC was conducted to restore airway patency. This indicated that treating tracheal tumors with APC may be an effective type of palliative therapy to provide immediate relief, as well as long-term improvements in patient quality of life. Although it is useful to combine palliative measures with therapeutic agents, including radiation and biological or chemical agents, the current treatment strategies are inadequate. For example, radiotherapy may be an effective method to locally control tracheal melanoma, however, it does not improve long-term survival (16).

In conclusion, the patient in the current case was treated with radiation and dacarbazine-based chemotherapy, which is a standard chemotherapeutic agent for malignant melanoma. Initially, the radiotherapy facilitated with controlling the local spread of the tumor, however, follow-up CT scans revealed distant metastasis to the lungs. In future, targeted cancer therapies using molecules, such as BRAF inhibitors, and cytotoxic T-lymphocyte-associated protein, programmed cell death protein 1 and programmed death-ligand 1 antibodies (17-20), may be effective options for treating cases of advanced malignant melanoma, including those originating in the trachea.

\section{Acknowledgements}

The authors would like to thank Ms. Mutsumi Yamazaki, Mr. Taiki Miyauchi, Dr Kazushige Wakuda, Dr Takuya Oyakawa, Dr Yasushi Hisamatsu and Dr Hiroaki Akamatsu for their assistance in preparing this case report.

\section{References}

1. Urdaneta AI, Yu JB and Wilson LD: Population based cancer registry analysis of primary tracheal carcinoma. Am J Clin Oncol 34: 32-37, 2011

2. Rostom AY and Morgan RL: Results of treating primary tumours of the trachea by irradiation. Thorax 33: 387-393, 1978.

3. Maziak DE, Todd TR, Keshavjee SH, Winton TL, Van Nostrand P and Pearson FG: Adenoid cystic carcinoma of the airway: thirty-two-year experience. J Thorac Cardiovasc Surg 112: 1522-1532, 1996.

4. Li W, Ellerbroek NA and Libshitz HI: Primary malignant tumors of the trachea. A radiologic and clinical study. Cancer 66: 894-899, 1990.

5. Lens MB and Dawes M: Global perspectives of contemporary epidemiological trends of cutaneous malignant melanoma. Br J Dermatol 150: 179-185, 2004.

6. Duarte IG, Gal AA and Mansour KA: Primary malignant melanoma of the trachea. Ann Thorac Surg 65: 559-560, 1998.

7. Terra RM, Minamoto H, Junqueira JJ, Falzoni R, Pego-Fernandes PM and Jatene FB: Tracheal malignant melanoma: successful outcome with tracheal resection. Ann Thorac Surg 86: 308-310, 2008.

8. Nattout M, Fuleihan N, Sabra O, Aburizk I and Hamdan AL: Primary malignant melanoma of the trachea. Middle East J Anesthesiol 20: 607-609, 2010.

9. Cekin E, Cincik H, Yilmaz I and Gungor A: Primary malignant melanoma of the trachea: case report. Ear Nose Throat J 89: E18-E20, 2010.

10. Nureki S, Miyazaki E, Fujisaki H, Ito T, Kumamoto T, Tokuishi K and Kawahara K: Incidentally discovered primary malignant melanoma of the trachea. Intern Med 51: 1743-1746, 2012 .

11. Koh HK: Cutaneous melanoma. N Engl J Med 325: 171-182, 1991.

12. Jennings TA, Axiotis CA, Kress Y and Carter D: Primary malignant melanoma of the lower respiratory tract. Report of a case and literature review. Am J Clin Pathol 94: 649-655, 1990.

13. Colby TV, Koss MN and Travis WD: Tumors of the lower respiratory tract. In: Atlas of Tumor Pathology. Rosai J and Sobin LH (eds). 3rd edition. Armed Forces Institute of Pathology, Ed. Armed Forces Institute of Pathology, Washington DC, 483-487, 1995.

14. Jensen OA and Egedorf J: Primary malignant melanoma of the lung. Scand J Respir Dis 48: 127-135, 1967.

15. Capaccio P, Peri A, Fociani P, Ferri A and Ottaviani F: Flexible argon plasma coagulation treatment of obstructive tracheal metastatic melanoma. Am J Otolaryngol 23: 253-255, 2002. 
16. Lentsch EJ and Myers JN: Melanoma of the head and neck: current concepts in diagnosis and management. Laryngoscope 111: $1209-1222,2001$.

17. Ascierto PA, Minor D, Ribas A, Lebbe C, O'Hagan A, Arya N, et al: Phase II trial (BREAK-2) of the BRAF inhibitor dabrafenib (GSK2118436) in patients with metastatic melanoma. J Clin Oncol 31: 3205-3211, 2013.

18. Hamid O, Robert C, Daud A, Hodi FS, Hwu WJ, Kefford R, et al: Safety and tumor responses with lambrolizumab (anti-PD-1) in melanoma. N Engl J Med 369: 134-144, 2013.
19. Ott PA, Hodi FS and Robert C: CTLA-4 and PD-1/PD-L1 blockade: new immunotherapeutic modalities with durable clinical benefit in melanoma patients. Clin Cancer Res 19: 5300-5309, 2013.

20. Wolchok JD, Kluger H, Callahan MK, Postow MA, Rizvi NA, Lesokhin AM, et al: Nivolumab plus ipilimumab in advanced melanoma. N Engl J Med 369: 122-133, 2013. 